\title{
Lopinavir Therapy for COVID-19
}

\section{Dr.Dhanush Delish}

Medical Intern, Department of Community Medicine, Jawaharlal Nehru Medical College, Datta Meghe Institute of Medical Sciences (DU), Sawangi (Meghe), Wardha, Maharashtra, Pincode-442001

Email address: dhanushdelish123@gmail.com

Review Article

Conflict of Interest: None

\begin{abstract}
:
A dangerous respiratory ailment (COVID-19) because of serious intense respiratory condition severe acute respiratory illness causing Corona illness was first depicted in December 2019 at Wuhan (China), quickly turning into a worldwide disease. In the principal stage, when the duplication of virus assumed into a urgent pathogenic job, antiviral medications should have been pivotal on restricting viral-initiated visceral harm. Shockingly, no possible antiviral drug demonstrated viability for (Corona disease), and a few medications have been redepeloyed shall possibly confront the most sensational worldwide spreading disease. This paper shall audit the investigations assessing how lopinavir or ritonavir ally in utilisation againstCorona disease and already in severe acute respiratory disease and Middle Eastern respiratory disorder (MERS). I looked through many article to recognize all important clinic based and research center investigations distributed up to 15 May 2020; the rules on the utilization of in Corona disease were further straightforwardly looked on the site of the fundamental worldwide logical social orders and organizations. Accessible proof is right now scant and are of inferior standards. The proposals given for Corona disease fluctuate from its place plainly in opposition towards utilization of lopinavir alternatively ritonavir to different places those which are more substantial. As we would like to think, regardless of the questionable consequences of a significantrandomized clinical endeavor, and a few suggestions, clinicians ought not forsake the utilization oflopinavir alternatively ritonavir cure for (Corona disease), perhaps utilizing these medication through an imminent aimless endeavor, hanging tight for the aftereffects of the various progressing struggles assessing its viability.
\end{abstract}

Key words: Viability for pandemic, Middle East respiratory disorder (MERS), COVID-19, Lopinavir (LPV/r)

\section{Introduction}

We led a aimless, supervised, open-mark primer including hospitalized grown-up patients with affirmed severe acute respiratory or corona disease contamination, which causes the respiratory disease Corona disease, and an oxygen immersion (Sao2) of $94 \%$ or less while they were breathing surrounding air or a proportion of the circulating oxygen (Pao2) to the division of respired oxygen (Fio2) of under $300 \mathrm{~mm} \mathrm{Hg}$. Patients were haphazardly appointed in a 1:1 proportion to get either lopinavir-ritonavir (400 mg and $100 \mathrm{mg}$, separately) two times per day for 14 days, notwithstanding standard consideration alone. The essential end point was the opportunity to clinical improvement, characterized as the time from randomization to either an improvement of two focuses on a sevenclass ordinal scale or release from the emergency clinic, whichever started things out antiretroviral protease inhibitor action of Lopinavir utilized alternatively Ritonavir (promoter) in acute immune-deficiency syndrome contamination treatment with anticipation. Lopinavir is a peptide mimetic aspartate protease inhibitor of aids type 1 drug it demonstrates through official action on its reactant area, in this manner, forestalling the discontinuity of polyprotein of virus forerunners bring on about, utilitarian aminoacidwhich is important for virus reproduction. Lopinavir is generally added and mixed along with short promoter portions on ritonavir that seem to ameliorateuponThe study of the action of drugs in the body and rate of excretion of Lopinavirthrough easing back its digestion through hepatic route by the hindrance on cytochrome P450 3A chemical.[1] 
The most regularly suggested measurements of Lopinavir in grown-ups is $800 \mathrm{mg}$ day by day on additionof about 200 milligram dosage Ritonavir, typically in partitioned dosages. minute level aminotransferase in the plasma rises happens at an extent onvictims inside in about two months, typically asymptomatic and self-restricting with continuation of the drug; be that as it may, deadly cases have been accounted for .

Lopinavir which is created towards hindrance of aids proteolysis, the principle distinction as for the severe acute respiratory diseased partner exists in different contrasted multidimensional structure of the acquired immune deficiency syndromic aspartic proteolytic enzyme a and 3CLpro cysteine proteolytic enzyme,shall influence restraint effectiveness .

As of late, it has been called attention to by Baldelli et al. which shall build up ideal measurements of Lopinavir or ritonavir in Corona disease can end up significant, in spite of the fact that it has been totally neglected up until this point: correspondingly those who were medicated on hydroxychloroquine, every Lopinavir restorative medication checking brought about qualities over the helpful reach, and the TDM information investigation indicated the corona illness affectedvictims had Lopinavir fixations 3overlap inflated as contrasted and the aids patients. Basically every single examples surpassed edge centralization of 7000 $\mathrm{ng} / \mathrm{mL}$, famously connected scant bearableness in aids victims. Focal action both ofLopinaviralternatively Ritonavir mightget clarified by the severe acute respiratory illness and corona instigated hepatic harm, modified articulation of medication using chemicals, alongside likely cooperation with oxichloroquine. The creators astutely propose examinationwhich justified diminished portion of Lopinavir alternatively ritonavir
(400/100 mg every day) tweaking thecure bearableness in corona illness.

\section{Methods}

patients were surveyed for qualification based on constructive converse transcriptase polymerase chain response examine (sansure biotech) for severe acute respiratory illness and corona disease respiratory test at an area tried by nearby Center for Disease Control or by an assigned analytic research facility. Men and fertile women 18 years of age or more established were qualified in the event a demonstrative example that they had that was effective on polymerase chain reaction test, had pneumococcal pneumonia affirmed by chest xray, and had oxygenlevels (Sao2) of $94 \%$ or less during respiration process encompassing wind or proportion of the incomplete weight of partial pressure oxygen to the part of propelled oxygen (Fio2)/(Pao2:Fio2) at or under 300 millimeter of mercury. Rejection measures incorporated doctor choice whose contribution on the preliminary was not in the victim's wellbeing, existence of any situationwhichshall not grantsmooth and secure conductance of convention , familiar sensitivity otherwise touchiness to lopinavir/ritonavir, seems to show extreme hepatic sickness (e.g., liver cirrhosis, which clinically manifestsAAT levels of more than $5 \times$ the maximum furthest reaches than that under normal circumstance or an aspartic aminotransferase level more than $5 \times$ with furthest reaches than the typical reach), utilization of prescriptions which are not supposed to prescribed due to harmful effects with lopinavir alternativelyritonavir and which couldn't be supplanted without choice quit in the course of the time for testing; pregnancy or bosom taking care of, or known HIV contamination, due to worries about the advancement of protection from lopinavirritonavir whenever utilized without joining 
with different antiretrovirals. Patients who couldn't swallow got lopinavir or ritonavir with assistenceof a tube passing down from nasal area to gastric area. [2]

\section{Design and Oversight}

This was of an open-named, separately recombined, supervised preliminary directed from eighteenth of January year 2020, through third of February year 2020 (the time of enlistment of the final victim), at Jin Yin Tan medical center situated at Wuhan near Hubei province . during ongoing sight of crisis idea about the preliminary, fake treatments of lopinavir as another option ritonavir haven't beenprepared. Qualified test subjectswere haphazardly allotted in a 1 is to 1 proportion to get either lopinavir as anotheroption ritonavir $(400 \mathrm{mg}$ and 100 $\mathrm{mg}$, orally; uninhibitedly given by the public wellbeing jurisdiction) two times every day, in addition to standard consideration, or worthwhile consideration lone, for fourteendays. WorthfulQuality consideration involved, as essential, supportive oxygen, unintrusive and intrusive externally induced breathing , anti-toxin specialists, vessel constrictors uphold, nephritic-substitution treatment, and outside the body film ventilation .To adjust the dispersion of air upheld in middle of the two gatherings as a marker point of serious of respirational disappointment, random allocation was separately based on ventilatory help strategies at time of enlistment: no backed up by oxygen or whiff of air uphold with help of nasal pipe without choice cover, or high-stream puff of air, unintrusive ventilation, or obtrusive respirational assistance that includesExtracorporeal membrane oxygenation. The permutation of (four patients for each square) random allocation arrangement, that includes definition, had been set up with help of an analyst not associated with the prefatory, utilizing statistical analysis systematic programming, To limit portion predisposition, they conducted designation camouflage along with intuitive internet based reaction framework until randomallocation was done through the framework using PC alternatively telephone. [3]

The preliminary was endorsed with help of conventional surveys leading group of Jin Yin Tan medical facility. Composed educated assent was received from all test subjects or from the test subjects lawful agent if the victim was too sick to considereven providing assent. The prefatory was led by the standards of the Helsinki Declaration and the Clinical Practice orderson the Harmonization during International Conference. The creators were accountable for arrangement the prefatory and for instructions and breaking down the information. The creators vouch for the culmination and precision of the info and for the adherence of the primer to the conference. Full insights regarding the preliminary plan are given in the convention

\section{Clinical and Laboratory Monitoring}

Patients were evaluated once every day via prepared medical caretakers utilizing journal cards that caught information on a 7-classification ordinal scale and on wellbeing for about 28 days long, emergency clinic release, or passing. Wellbeing had been checked by the Good Clinical Practice office located Jin Yin tan medical center. Other clinic oriented information were videotaped utilizing the World Health Organization International Severe Acute Respiratory and Emerging Infections Consortium case narrative structure.16 oropharyngeal swab tests were received on the first day (preceding lopinavir alternatively ritonavir was controlled) and on fifth, tenth, fourteenth, twenty one and twenty eighth day till release or passing would have happened and were tried at Clinical Research Laboratory of teddy (Tigermed-DiAn 
Joint Venture), utilizing quantitative continuous Realtime reverse transcriptase reaction .ribonucleic acid was removed out of clinic oriented examples with the MagNA Pure 96 framework, identified and measured by Cobas Z480 QPCR (Roche), on about utilization of Light Mix Modular Severe Acute Respiratory and Emerging Infections corona illness tests all These examples arereceived from each of the hundred and ninety nine test subjectsof thosewho are as yet alive at each point of time. Testing didn't stop when the swab eventuated to a negative result. Patterned swabs from throathad been tried for recognition of E-quality, RDRP quality, and $\mathrm{N}$-quality, and looking in on tests on the ensuing were fathomably and subjectively recognized for E-quality. Clinic based information were catalogued at case records in paper structures and afterward twofold data set went onto an electron deviceand approved next to preliminary personnel. [4]

\section{Measures}

the time toward The essentially required end was directed was towardsclinical improvement keptin mind, characterized as the schedule fromrandomized allocation to both the focuses towards its advancement (from the position at randomized allocation) with help of 7-classification statistic scale or live releasedthat was residing at the health centre, whichsoever started things out. The last purpose of clinic basedadvancementwere utilized on part of our past flu study and was additionally suggested by the world health organizationresearch and developmentlayout master batch. Ordinal scales, 18 of them had been utilized even as final focuses through clinic based preliminaries in victims bedridden with extreme Influenza. The seventh-class type of Ordinal scale sixteen to nineteen of them comprised about below mentioned classifications: 1.Those who resumed ordinary activities and are not bedridden;
2. not bedridden, yet unfit on continuing typical exercises; 3.Admitted to hospital, with no need of additional auxiliaryoxygen; 4.Those bedridden , in need of additional auxiliary oxygen; 5.Those who are admitted to hospital, requiring high concentration Oxygen treatment through nasal cavity, unintrusive assisted manual external respiration or even both; 6.victims who areHospital admitted , required extra corporeal mechanical oxygenation, intrusive no assisted external respiration, or together; and 7. demise. [5]

Clinically other results includingpositions of clinical scenariowas evaluated with help of 7 -classification seventh and fourteenth day on ordinal scale, $28^{\text {th }}$ day of fatality rate, the span of manually assisted external respiration, the term of survivors who were admitted to hospital, and starting from the schedule (in days) of therapy inception to victims demise. measuresagainst virological status incorporated extents of ribonucleic acid of virus discovery during the long run and ribonucleic acid of virus titre zone under the bend (area under curve) estimations.

Wellbeing results added unfriendly occasions that happened during ongoing therapy, genuine antagonistic occasions, and untimely cessation of treatment. Antagonistic occasion was ordered adverse effect by the National Cancer Institute Common Terminology Criteria , adaptation 4 .

\section{Analysis}

During the start preliminary were in fast reaction to the corona illness general wellbeing crisis, at the course of which there had been extremely restricted data on clinic based results in hospital admitted patients with corona illness. The first complete example size had beencoordinated at 160 , as it shall furnish the preliminary with eighty percentage capacity to recognize a distinction, at a both biased response importance level of 
$\alpha=0.05$, of eight days in the middle opportunity to clinic based advancement between both the gatherings, expecting that the middle standardizing time at center care bunch was twenty days and that of seventy five percentage of the test subject would arrive at clinic based advancement. The arranged enlistment of hundred and sixty victims at the preliminary happened rapidly, evaluation done by them at the time was a preliminary which had beenlow powered ; consequently, the choice had to bepreparedtowards proceeding with enlistment of examiners. Therefore, another specialistwho had (Remdesivir) opened up towards clinic based preliminaries, for which we selected to turn down enlistment on these preliminaries.[6]

Essential viability investigation was with a goal aiming to heal the premise and incorporated every patient whosoever did go through randomized allocation. This opportunity to clinic based advancement was surveyed after every victims that had arrived at $28^{\text {th }}$ day of the month, though unable to arrive at clinic based advancement or passing before $28^{\text {th }}$ day of the month considering if it is rightlyedited at $28^{\text {th }}$ day (correct-controlling happens only if the occasion might have happened at the end of the final time an individual were perceived, while the occasion planned at that time was shrouded with opaqueness). Kaplan-Meier plotted the log rank test after which he came to a conclusion that there is still a contrast place for improvements.through the Cox relative peril method danger proportions were concluded to be around $95 \%$.the bunch of five victims who had been referred to lopinavir alternatively ritonavir didn't get any dosages (three of them kicked the bucket inside 24 hours) yet were remembered for the aim to-treat investigation, since no proportional evacuations happened in the standard-care gathering.the adjusted aim to-heal examination which prohibited 3earliest passing's were additionally achieved. after hoc investigations incorporate the subgroup examination for NEWS (National Early Warning Score 2) mentioned underneath alternatively more prominent than five and the individuals which went through randomized allocation as long as twelve days or even more than twelve days subsequentlythe first symptom of illness.

Since measurable investigation had excluded an arrangement for adjusting for variety in tests for auxiliary or different results.Results are accounted for as reference gauge points and with about $95 \%$ of certainty stretches. For variety purposes the width of certainty stretch had not been hampered with ,time durationhaven't been utilized on deriving conclusive management impacts towards optional results. Wellbeing investigations depended on the patients' genuine treatment introduction. Factual investigations were led with SAS programming, rendition 9.4 (SAS Institute).[7]

\section{Outcome of the study}

All randomly chosen genuinely comorbid patient infected with corona illness treated with lopinavir alternatively ritonavir therapy did not show clinical based advancement inhealth or change fatality rate, not quite the same as that related with standard consideration alone. In any case, in the changed aim towards treating investigation, on which we avoided victims with early passing (3), amongst the bunch distinction at middle chance towards clinic based advancement were critical, yet unassuming. off record, general fatality rate in this preliminary lower about $11 \%$ to $14.5 \%$ in contrast to preliminary which had improved to $22.1 \%$ fatality announced on an introductory unmistakable investigations victims who were admitted to hospital for corona illness that portrays gravely ill test groups the study have chosen. 
The selection of patient in population were heterogenous for the duration and seriousness of the disesase enlisted. The hoc subgroup investigated afterwards, difference between those underwent lopinavir alternatively ritonavir therapy to normal care group the normal care group was mathematically found to be more better among the all patient who were treated 12 days later to the appearence of the first symptom as to those who were treated on the later stages.Lopinavir alternatively ritonavir therapies apparent restorative property on prior stage treatment to patient needs to be examined further through further studies. Those who were affected with either Severe acute respiratory illness or viral pneumonia or influenza infection the treatment of lopinavir alternatively ritonavir therapy seems to have maximum impact in the second week of antiviral treatment as compared to the other weeks of treatment that too in the early initiation of the therapy just next to the first symptom appeared and also it is more helpful in contrast to the patient in assisted external respiration or with renal failure and other contaminations. The speculated perceptionsrequires around long duration of extra examinations and decisionwhether lopinavir alternatively ritonavir therapy administered at a specific phase.During phase ailment can lessen a few entanglements in corona illness. Addition of lopinavir alternatively ritonavir therapy diminish ribonucleic acid of virus burden or even term of ribonucleic acid of virus perceptibility contrasted and through strong consideration without help. Severe acute respiratory illness or corona illness ribonucleic acid structure was as yet distinguished in 40.7percentage of the patients in the lopinavir-ritonavir bunch at end of the preliminary (day 28).report indicates virus sheds its progeny from its shell the $20^{\text {th }}$ days onwards uptil $37^{\text {th }}$ day of the month. Neither that review nor the present reviewdiscovered proof that lopinavir alternativelyritonavir applied a huge impact towards eradicating the corona virus . it is seriously doubtful about the poor efficacy against viral antigen.however the inspecting techniques utilized at present preliminary was in all probability imperfect. Tests were taken just irregularly (on days 1, 5, 10, 14, 21, and 28), and more regular inspecting in the initial 5 days might have given more point by point portrayal of viral burden energy in the two gatherings over this basic period. Also, past examinations have indicated swab of cotton sampled from throat examples had low virus loads than nasopharyngeal samples and significantly, analysis could not be done of respiratory tracts lower portion plot emissions. Off record, contingent upon type of cell utilized, the half compelling focuses (EC50) of lopinavir artificial environment outside living organism for severe acute respiratory illness corona illness have reduced from 4.0 to 10.7 microgram per milliliteralbeit different investigations detailed that lopinavir was inactive 26 or that higher fixations ( $25 \mu \mathrm{g}$ per milliliter) were needed for inhibition.7 For MERS$\mathrm{CoV}$, the EC50 values have gone from 5 to roughly $7 \mu$ g per milliliter). $1,8,13$ Both the mean pinnacle $(9.6 \mu \mathrm{g}$ per milliliter) and box (5.5 $\mu$ g per milliliter) serum groupings of lopinavir in grown-ups simply approach these fixations. Regardless of whether the EC50 esteem is a satisfactory limit and whether unbound lopinavir fixations in human plasma are adequate for restraint of SARS-CoV-2 are questionable.

Lopinavir alternatively ritonavir beneficiaries of around 14 percentage couldn't finish complete a total of 2 week course of therapy. The reason behind failure in course of action of completion of therapy was due to life threatening side effects of inability to eat due to gastritic pain,hepatic injury , pancreatic inflammation, extreme dermatitis infectious disease manifestation even QT prolongation and at last numerous 
medication associations because of CYP3A restraint, are very much recorded with this medication blend. The result profile saw in the current preliminary excites worry on utilization of higher or more delayed lopinavir alternatively ritonavir portion course on an attempttowardsdeveloping results.[8]

Our preliminary has a few impediments. Specifically, the preliminary was not blinded, so it is conceivable that information on the treatment task may have impacted clinical dynamic that might have influenced the ordinal scale estimations we utilized. We will keep on after these patients to assess their drawn out anticipation. The qualities of the patients at benchmark were commonly adjusted over the two gatherings, however the fairly higher throat viral burdens in the lopinavir-ritonavir bunch raise the likelihood that this gathering had more popular replication. In spite of the fact that we didn't notice contrasts between bunches in the recurrence of utilization of simultaneous pharmacologic intercessions, for example, glucocorticoids, this may have been another confounder. What's more, around $45 \%$ and $40 \%$ of the patients in lopinavir-ritonavir bunch had positive RNA recognition by throat swabs on day 14 and day 28, separately, yet we don't have the foggiest idea whether irresistible infection was as yet present, since we didn't endeavor infection disconnection or survey the conceivable development of SARS-CoV-2 variations with decreased vulnerability to lopinavir. At last, we don't have information on the lopinavir introduction levels in these truly and frequently fundamentally sick patients[9]. Few of the related studies on indigenous treatment modalities were reviewed[10$15]$.

\section{Conclusion}

Taking everything into account, we found that lopinavir-ritonavir treatment didn't fundamentally quicken clinic based advancement in health, increase fatality, otherwise reduce throat ribonucleic acid of virus perceptibility on victims affected by genuine Corona illness. The review of these studies ought to illuminate future surveys and investigations on this particular drug along with upcoming drugs against those who were contaminated with severe acute respiratory syndrome corona illness. Regardless of whether consolidating lopinavir-ritonavir with other antiviral specialists, as has been done in SARS5,20 and is being concentrated in MERS-CoV,15 may upgrade antiviral impacts and improve clinical results stays to be resolved.

\section{References:}

1. Yao T.T., Qian J.D., Zhu W.Y., Wang Y., Wang G.Q. A systematic review of lopinavir therapy for SARS coronavirus and MERS coronavirus-A possible reference for coronavirus disease-19 treatment option. J. Med. Virol. 2020;92:556-563. doi: 10.1002/jmv.25729.

2. Ford N., Vitoria M., Rangaraj A., Norris S.L., Calmy A., Doherty M. Systematic review of the efficacy and safety of antiretroviral drugs against SARS, MERS or COVID19: Initial assessment. J. Int. AIDS Soc. 2020;23:e25489. doi: 10.1002/jia2.25489.

3. Zhong H., Wang Y., Zhang Z.L., Liu Y.X., Le K.J., Cui M., Yu Y.T., Gu Z.C., Gao Y., Lin H.W. Efficacy and safety of current therapeutic options for COVID-19 -lessons to be learnt from SARS and MERS epidemic: A systematic review and meta-analysis. Pharmacol. Res. 2020;157:104872. doi: 10.1016/j.phrs.2020.104872.

4. Lu H. Drug treatment options for the 2019-new Coronavirus (2019nCoV) Biosci. Trends. 2020;14:69-71. doi: 10.5582/bst.2020.01020. 
5. Kupferschmidt K., Cohen J. Race to find COVID-19 treatments accelerates. Science. 2020;367:1412-1413. doi: 10.1126/science.367.6485.1412.

6. "Solidarity" Clinical Trial for COVID-19 Treatments. [(accessed on 6 May 2020)]; Available online: https://www.who.int/emergencies/d iseases/novel-coronavirus2019/global-research-on-novelcoronavirus-2019-ncov/solidarityclinical-trial-for-covid-19treatments.

7. Baldelli S., Corbellino M., Clementi E., Cattaneo D., Gervasoni C. Lopinavir/ritonavir in COVID-19 patients: Maybe yes, but at what dose? J. Antimicrob. Chemother. 2020:dkaa190. doi: 10.1093/jac/dkaa190.

8. Yousefifard M., Zali A., Mohamed Ali K., MadaniNeishaboori A., Zarghi A., Hosseini M., Safari S. Antiviral therapy in management of COVID-19: A systematic review on current evidence. Arch. Acad. Emerg. Med. 2020;8:e45.

9. Chan K.S., Lai S.T., Chu C.M., Tsui E., Tam C.Y., Wong M.M.L., Tse M.W., Que T.L., Peiris J.S.M., Sung J., et al. Treatment of severe acute respiratory syndrome with lopinavir/ritonavir: A multicentre retrospective matched cohort study. Hong Kong Med. J. 2003;9:399406.

10. Parwe, S.D., M.A. Nisargandha, and R. Thakre. "Role of Convalescent Plasma Therapy in New Coronavirus Disease (Ncovid19): A Review." International Journal of Research in Pharmaceutical Sciences 11, no. Special Issue 1 (2020): 546-49. https://doi.org/10.26452/ijrps.v11i SPL1.2846.

11. Singh, A.K., M. Yeola, and N. Singh. "A Review of Convalescent
Plasma Therapy an Immediately Available Therapeutic Option for Covid19 in Light of the Available Data." Journal of Datta Meghe Institute of Medical Sciences University 15, no. 1 (2020): 14952.

https://doi.org/10.4103/jdmimsu.jd mimsu_149_20.

12. Andhare, R., S. Muley, and S. Bhirange. "Ayurvedic Perspective of COVID-19 Diagnosis and Management." Journal of Critical Reviews 7, no. 8 (2020): 1070-72. https://doi.org/10.31838/jcr.07.08.2 23.

13. Vaidya, H.D., and P. Sawarkar. "Ayurvedic Remedies for COVID19 - A Conceptual Study." International Journal of Research in Pharmaceutical Sciences 11, no. Special Issue 1 (2020): 286-91. https://doi.org/10.26452/ijrps.v11i SPL1.2714.

14. Thakre, P.P., V. Ade, and S. Parwe. "Review on Efficacy of Herbal Antiviral Drugs against COVID-19." International Journal of Research in Pharmaceutical Sciences 11, no. Special Issue 1 (2020): 1116-21. https://doi.org/10.26452/ijrps.v11i SPL1.3552.

15. Dhole, P.D., V.K. Lohe, R.P. Kadu, S.C. Mohod, M. Meshram, and G.A. Thakare. "Post COVID19 Protocol of Treatment, Radiologic Examination and Infection Control in Dentistry." International Journal of Research in Pharmaceutical Sciences 11, no. Special Issue 1 (2020): 1384-89. https://doi.org/10.26452/ijrps.v11i SPL1.3664. 